\title{
Comparison between numerical and experimental water-in-oil dispersion in a microchannel
}

\author{
Philippe Desjonquères ${ }^{1}$, Thibaut Ménard ${ }^{1}$, Dominique Tarlet ${ }^{* 2}$, Jérôme Bellettre ${ }^{2}$ \\ ${ }^{1}$ UMR 6614-CORIA, Technopôle du Madrillet, B.P. 12, Avenue de l'Université, 76801 Saint \\ Etienne du Rouvray Cedex, France \\ ${ }^{2}$ Laboratoire de Thermique et Energie de Nantes, LTEN UMR CNRS 6607,BP 50609, 1 rue \\ Christian Pauc, 44306 Nantes cedex 3, France \\ ${ }^{*}$ Corresponding author: dominique.tarlet@univ-nantes.fr
}

\begin{abstract}
The dispersion of water inside a flow of oil is investigated in a microfluidic device, producing a water-in-oil emulsion. The liquid-liquid flow mainly differs from those presented in existing literature through its high capillary number (between 3 and 14), and in the head-on collision between water and oil streams. By comparing with experimental data, numerical simulations can provide more information about the topology of the flow. A coupled Volume of Fluid and Level Set method (CLSVOF) is used to treat the interface between both phases and incompressible Navier-Stokes equations are solved. Three set of parameters, close to those in the experimental setup, are investigated to compare experimental and numerical results. The comparison between experiments and simulation provides a precise knowledge of the liquid-liquid dispersion process and the overall flow pattern within the microfluidic device.
\end{abstract}

\section{Keywords}

Microchannel, water-in-oil dispersion, liquid-liquid flow

\section{Introduction}

Liquid-liquid dispersion within microfluidic devices has become an important issue over the last decade [1]. An emulsion is defined as the temporarily stable dispersion of a liquid into another one that is not miscible [2]. When the scale of the liquid-liquid flow is smaller than its capillary length [3], interfacial tension dominates over shear stress and gravity [4], making the dispersion highly reproducible in slow conditions [5]. These slow conditions ensure a highly monodisperse emulsion [6], that is usually appropriate for targeted applications like microreaction synthesis [7]. However, other application like high flow-rate biofuel production [8] benefit from the considerably increased surface-to-volume ratio $[9,10]$ of microfluidic liquid-liquid dispersion.

In order to better understand the physics of microfluidic in high flow-rate liquid-liquid dispersion, experimental results of the obtained mean diameter and liquid-liquid flow photographies [8] are compared to numerical results. At the present stage, a quantitative validation of the model is not obtained, but we present a qualitative comparison of the liquid-liquid flow pattern. In a first part, experimental material and methods are presented, then numerical methods used to investigate such flows are briefly detailed. Finally, first comparisons are discussed.

\section{Material and methods}

The experimental facility is designed to produce high flow-rate water-in-oil flow within a microfluidic device. The capillary number $\mathrm{Ca}$ (Eq. 1) represents the ratio between shear stress ( $\mu \mathrm{V} / \mathrm{L})$ and interfacial tension $\sigma_{\mathrm{wo}} / \mathrm{L} . \mu$ and $\mathrm{V}$ are the viscosity and the superficial velocity of the continuous phase, e.g. filtered sunflower oil in thiscase. It reaches values between 2 and 14, which is three orders of magnitude higher than most of the situations investigated in scientific literature [11].

$$
\mathrm{Ca}=\mu \mathrm{V} / \sigma_{w o}
$$

The microfluidic device shown Fig. 1 enables a finely dispersed emulsion due to three main reasons. First, streams of oil and water are faced in a head-on collision. This maximizes the energy available to fractionate the water stream, since kinetic energies are added in the collision. Secondly, the viscosity $\mu$ is very high (up to 52.2 $\mathrm{mPa}$.s) to maximize shear stress from the continuous phase. Thirdly, the water inlet is smaller than the oil inlet to produce a swirling flow downstream the impinging zone. Such a swirling flow enhances curvature of streamlines, and in turn shear-induced break-up. The oil inlet and the emulsion outlets are squared cross-sections, with 600 $\mu \mathrm{m}$ per side. The water inlet is also squared, with $300 \mu \mathrm{m}$ per side. All connections between pumps and mini- 
channel are made using Fluoropolymer (FEP) tubing with an internal diameter of $1.55 \mathrm{~mm}$. The outlets are at the atmospheric pressure.

The microchannel is made of two PMMA slabs screwed together to avoid leakage at high injection pressure up to 7 bar. The hydrophobic PMMA material [12] is widely used in the field of two-phase microfluidics, to avoid destruction of the flow structure due to wettability effects on the walls [1]. The average surface roughness on the etched surface at the bottom of the channel is $460 \mathrm{~nm}$, measured by means of an ALICONA optical profilometer. This roughness is very small, when compared with the size of the channels (between 200 and $600 \mu \mathrm{m}$ ), and cannot significantly disturb the flow. The interfacial tension was measured using a KRÜSS tensiometer K-12, since $\sigma_{\mathrm{wo}}$ is a crucial property to investigate the physics of shear induced breakup [11]. The temperature of oil and water is maintained at $25^{\circ} \mathrm{C}$ in a LAUDA thermostated bath, and measured by a thermocouple in the beakers and in the microfluidic device. The size of dispersed water droplets is measured in a sample of emulsion coming out from the microfluidic device using a ZEISS optical microscope. The sufficient size of the sample is determined to calculate the mean diameter of water droplets.

Two piston pumps (ARMENAPF-100-25-1) are used for supplying water and filtered sunflower oil at a high pressure (up to 5 bars). Flow rate measurements are performed using weighing scales (Sartorius-MSE $2203,1 \mathrm{~Hz}$ sampling) connected to a computer. The weighing scales have a measurement accuracy of $10^{-3} \mathrm{~g}$, which results in an uncertainty of $5 \%$ of the flow rate. The flow pattern is observed with a fast CCD camera LAVision HighSpeed Star 6 equipped with the macro objective, necessary to resolve the millimeter scale. The focus was made in the channel depth. The light source consists of a $50 \mathrm{~W}$ halogen spot located in the line of sight, beyond the transparent microfluidic device.

All the details about conception of the microchannel and the experimental set-up, including measurement uncertainties can be found in [8].
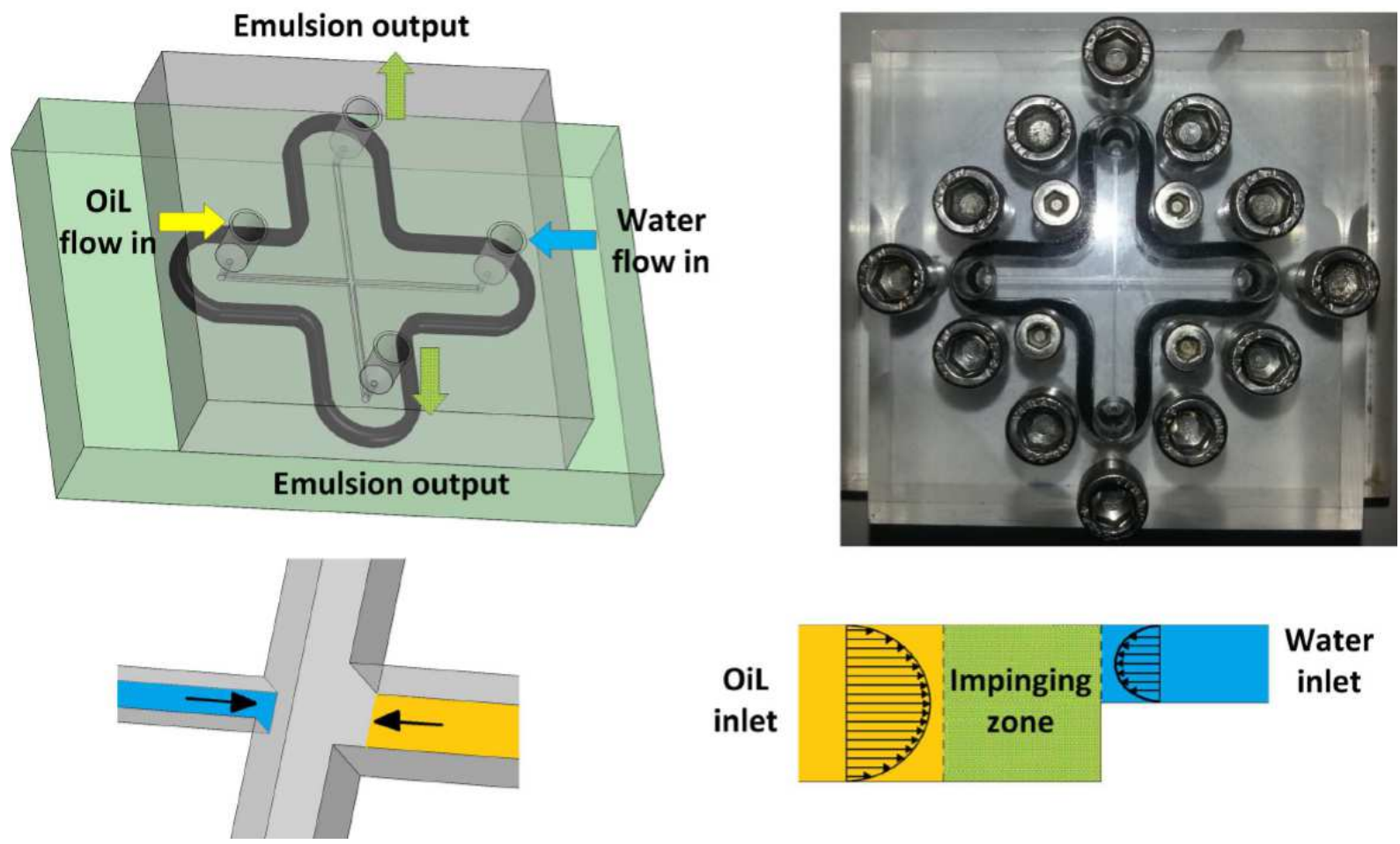

Figure 1.Microfluidic device : water and oil streams are faced in a head-on collision within the impinging zone.

The used fluids are tap water and oil with additives to decrease viscosity $\mu$ and interfacial tension $\sigma_{\text {wo: }}$ Butanol, and surfactant SPAN 83. Tab. 1 shows the three cases chosen for the present study : A, B and C with physical properties at $25^{\circ} \mathrm{C}$, as it was maintained in the experimental set-up. The capillary length $\mathrm{L}_{c}$ is also mentioned : it is always larger than the channel side so that interfacial tension dominates [4]. The obtained mean diameter $D_{d}$ shown in Tab. 1 is similar (between 20 and $21 \mu \mathrm{m}$ ) for cases $(A)$ and $(B)$, it is more than $50 \% \operatorname{larger}(32.48 \mu \mathrm{m})$ in case $(C)$. The effect of a lower viscosity of the continuous phase is a decreased shear stress exerted on the water phase, resulting in a larger mean diameter of water droplets. 
Table 1.Characteristics of the three cases chosen for study.

\begin{tabular}{|c|c|c|c|}
\hline Cases & A & B & C \\
\hline $\begin{array}{c}\text { Continuous phase } \\
\text { at } 25^{\circ} \mathrm{C}\end{array}$ & Pure oil & $\begin{array}{c}\text { Oil }+0.3 \% \text { wt. } \\
\text { SPAN 83 }\end{array}$ & Oil + 5\% wt. Butanol \\
\hline Qo $\left[\mathrm{mL} \cdot \mathrm{min}^{-1}\right]$ & 72.55 & 62.84 & 76.23 \\
\hline Qw $\left[\mathrm{mL} \cdot \mathrm{min}^{-1}\right]$ & 6.94 & 9.06 & 13.85 \\
\hline$\mu[\mathrm{mPa} \cdot \mathrm{s}]$ & 52.2 & 52.2 & 40.4 \\
\hline$\sigma_{\mathrm{wo}}\left[\mathrm{mN} \cdot \mathrm{m}^{-1}\right]$ & 27.6 & 11.7 & 32.48 \\
\hline $\mathrm{D}_{\mathrm{d}}[\mu \mathrm{m}]$ & 20.78 & 20.46 & 3.13 \\
\hline $\mathrm{L}_{\mathrm{c}}[\mathrm{mm}]$ & 4.6 & 2.99 & \\
\hline
\end{tabular}

\section{Numerical methods}

To simulate such flow, we use an in-house code generally applied for the study of liquid jet atomization [13]. The following incompressible Navier-Stokes equations are solved thanks to a projection method and coupled with interface transport equation performed by a CLSVOF method [14,13]:

$$
\frac{\partial \rho \boldsymbol{U}}{\partial t}+\boldsymbol{\nabla} \cdot(\rho \boldsymbol{U U})=-\nabla p+\nabla \cdot(2 \mu \boldsymbol{D})+\sigma \kappa \boldsymbol{n} \delta_{\Gamma}
$$

Surface tension force is treated as a jump condition for the pressure through a Ghost Fluid method [15]. The convective term is computed in a mass/momentum conserving framework [16,17], where one part comes from mass fluxes deduced by the VOF advection and the second part comes from a WENO5 interpolation. The diffusive term is computed thanks to the method developed by Sussman [18] and physical properties are expressed by the VOF or Level Set functions.

The main idea of the CLSVOF method is to benefit of both geometrical computations (normal and curvature of interface) from Level Set, and mass conservation from the VOF. The transport of the VOF is ensured by the method developed by Weymouth [19].

Due to the symmetry of the flow, only half of microchannel is computed and symmetric boundary condition is imposed to mimic the other part. Then, inlet boundary conditions for the water and oil are prescribed using a velocity profile of square channel and flow rates of experimental setup are imposed. Finally, no specific treatment is dedicated for the dynamic of triple line on wall boundary condition. Numerical domain is presented on the figure 2. This equivalent size is $(1800 \times 1800 \times 600) \mu \mathrm{m}^{3}$, that means water and oil inlet channel have a length of $600 \mu \mathrm{m}$, and the equivalent mesh size is $(384 \times 384 \times 128)$. 

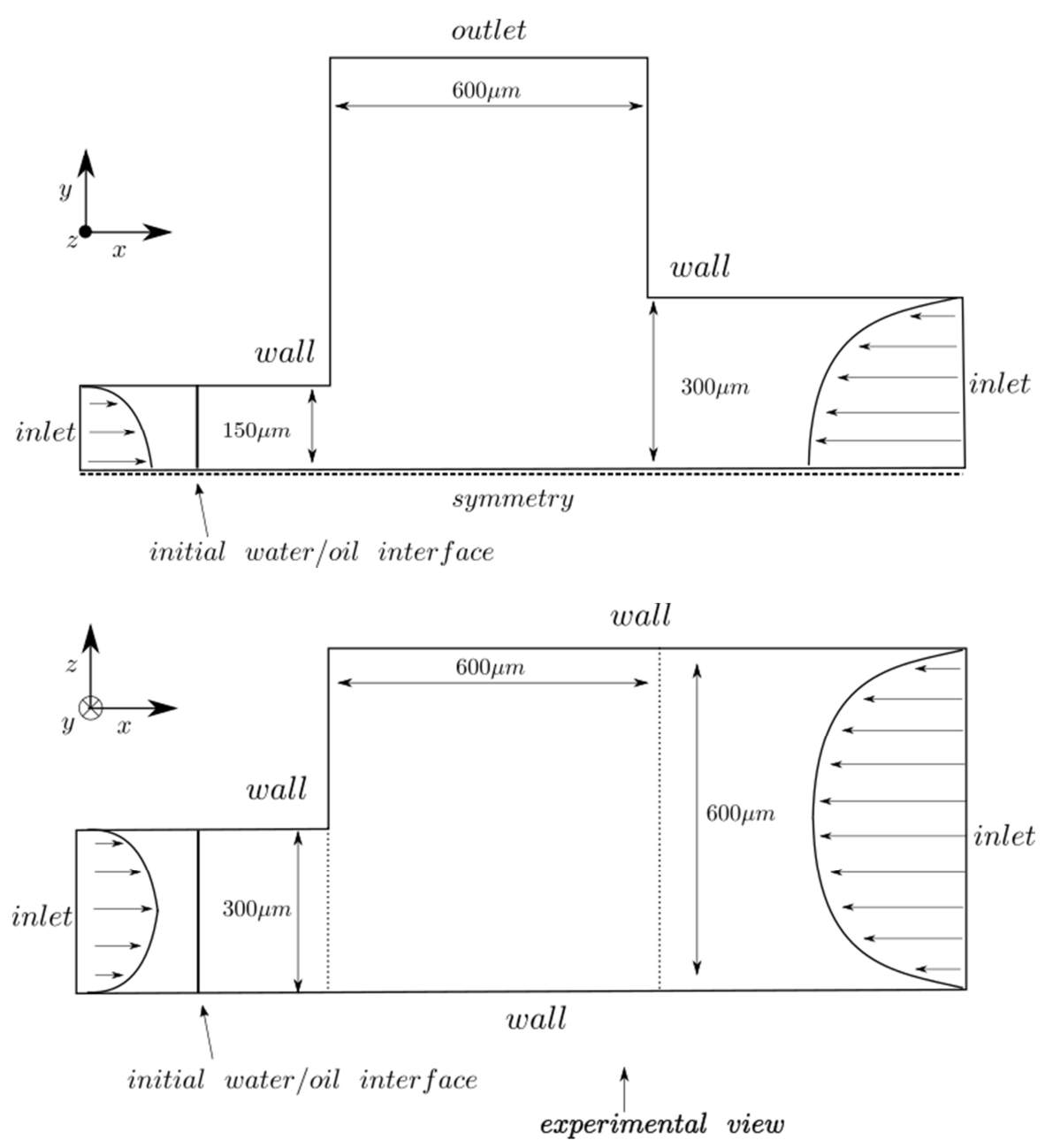

Figure 2.Computational domain.

\section{Results and discussion}

Fig. 3 shows the images from the CCD camera, within the impinging zone. In cases $(A)$ and $(B)$, where viscosity of the continuous phase is high ( $\mu=52.2 \mathrm{mPa}$.s), the water stream is limited in space. Whereas in case (C), which has lower viscosity $(\mu=40.4 \mathrm{mPa}$.s), the water phase invades nearly all the available space of the channel. In all cases, a symmetry of the liquid-liquid flow can be noticed downstream the impinging zone. A swirling flow can be seen, enhanced by the higher position of the water inlet.

On the Fig. 4 are reported numerical results of cases A, B and C compared to experimental images. We can observe that computations present thin structures at the exit of inlet water microchannel. The arc shape of these sheets seems to be similar to what is observed experimentally (case A and B). 


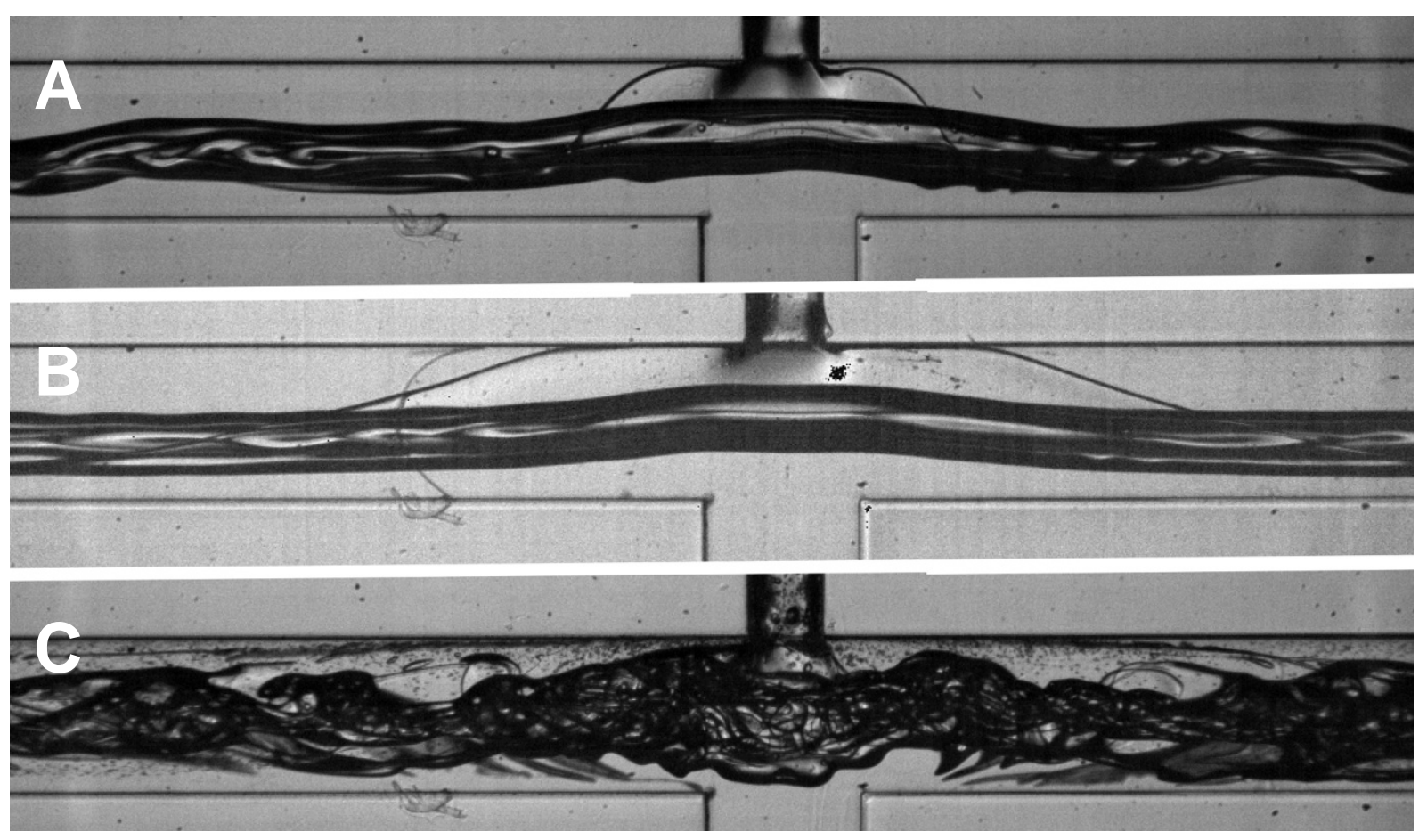

Figure 3.Image of the CCD camera in the impinging zone : Case A (top), B (middle) and C (bottom) - channel width : $600 \mu \mathrm{m}$.

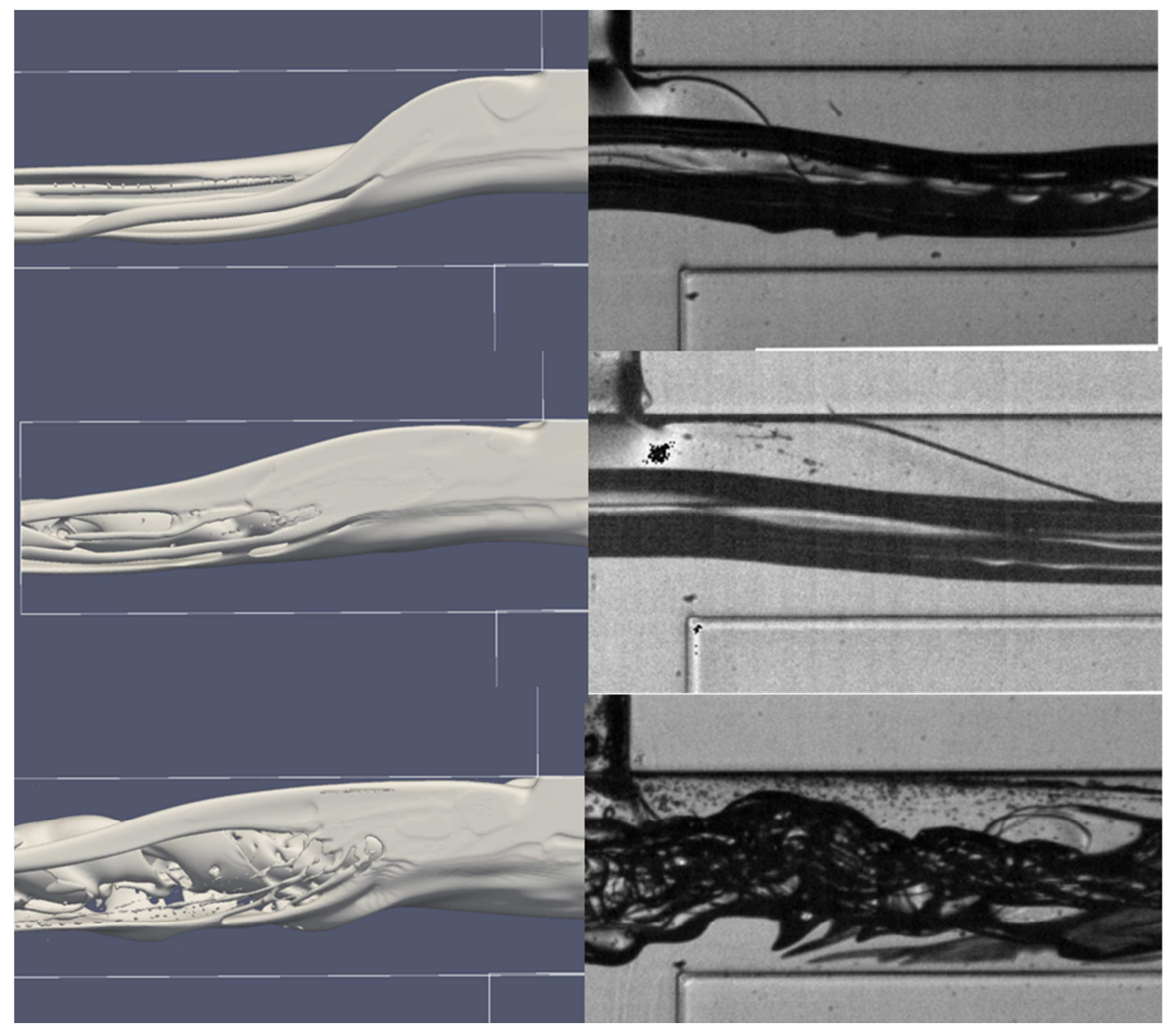

Figure 4.Experimental view of numerical results. (Top to bottom : cases A, B and C)

This work is licensed under a Creative Commons 4.0 International License(CC BY-NC-ND 4.0). 
Figure 5 reproduces some slices of case $A$ to show the structure of the flow in the impact zone and its consequences in the rest of the microchannel. We can see that the vortex formed by the impact of water/oil flow, subsist on the microchannel and then rolls up the interface. This behaviour, present in all cases, is responsible for the formation of very thin structures which are not well captured as shown by the formation of some holes in liquid sheets. These structures will probably influence the drop size distribution observed in the experiment at the end of the microchannel.
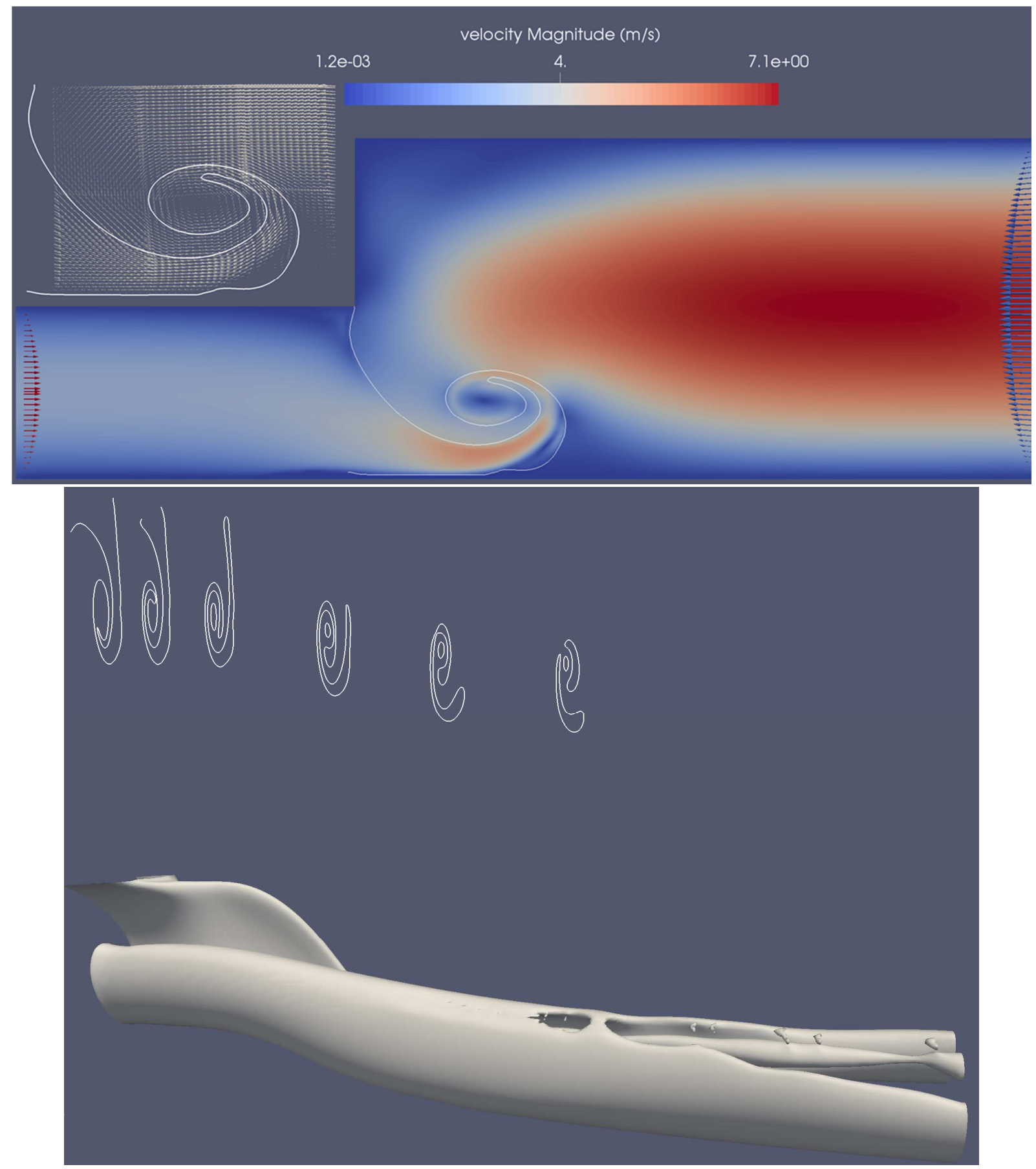

Figure 5.Structure of the flow (case A) on the impact zone and at different heights of the micro-channel coloured by velocity, from blue (slowest), to red (fastest). 


\section{Conclusions}

The comparison between experimental water-in-oil dispersion within a microchannel and numerical simulation are carried out qualitatively at this stage. Despite the observed differences, these results show the capabilities of numerical computation to capture and reproduce the main structure of the flow. They should easily be improved with a finest mesh. The triple line and contact angle dynamics is probably another parameter which can influence the numerical results.

Finally, to investigate the droplet size distribution, a longer computation domain has to be simulated.

\section{Acknowledgements}

Computations have been carried out in CRIANN (Centre Régional Informatique et d'Applications Numériques de Normandie).

\section{Nomenclature}

$\mathrm{D}_{\mathrm{d}} \quad$ mean water diameter $[\mu \mathrm{m}]$

$L_{c} \quad$ capillary length [mm]

$\mathrm{Q}_{w} \quad$ water flow rate $\left[\mathrm{mL} \cdot \mathrm{min}^{-1}\right]$

Qo oil flow rate $\left[\mathrm{mL} \cdot \mathrm{min}^{-1}\right]$

$\mu \quad$ oil viscosity [mPa.s]

$\sigma_{\mathrm{wo}} \quad$ interfacial tension $\left[\mathrm{N} \cdot \mathrm{m}^{-1}\right]$

\section{References}

[1] Zhao, C.-X., Middelberg, A.P.J., 2011, Chemical Engineering Science, 66, pp. 1394-1411.

[2] Engl, W., Backov, R., Panizza, P., 2008. Controlled production of emulsions and particles by milli- and microfluidic techniques. Current Opinion inColloid \& Interface Science 13 (13), pp. 206-216.

[3] Aarts, D., 2005. Capillary length in a fluid-fluid demixed colloid-polymermixture. J. Phys. Chem. B, 109, pp. 7407-7411.

[4] de Gennes, P.-G., Brochard-Wyart, F., Quéré, D., 2005. "Gouttes, bulles, perleset ondes". Belin.

[5] Engl, W., Backov, R., Panizza, P., 2008. Controlled production of emulsions and particles by milli- and microuidic techniques. Current Opinion in Colloid \& Interface Science 13 (13), pp. 206 -216.

[6] Sugiura, S., Nakajima, M., Iwamoto, S., Seki, M., 2001. Interfacial tensiondriven monodispersed droplet formation from microfabricated channel array. Langmuir 17 (1), pp. 5562-5566.

[7] Meng, Z.-J., Wang, W., Liang, X., Zheng, W.-C., Deng, N.-N., Xie, R., Ju, X.-J., Liu, Z., Chu, L.-Y., 2015. Plugand-play microfluidic systems from flexible assembly of glass-based flow-control modules. Lab Chip 15, pp. 18691878.

[8] Belkadi, A., Tarlet, D., Montillet, A., Bellettre, J., Massoli, P., 2015.Water-in-oil emulsification in a microfluidic impinging flow at highcapillary numbers. International Journal of Multiphase Flow 72, pp. 11-23.

[9] Burns, J. R., Ramshaw, C., 2001. The intensification of rapid reactions inmultiphase systems using slug flow in capillaries. Lab Chip 1, 10-15.

[10] Song, H., Tice, J., Ismagilov, R. F., 2003. A microfluidic system for controllingreaction networks in time. Angewandte Chemie International Edition 42 (7), pp. 768-772.

[11] Tice, J., Lyon, A., Ismaglov, R., 2004. Effects of viscosity on droplet formationand mixing in microfluidic channels. Analytica Chimica Acta 507, pp.73-77.

[12] Nisisako, T., Torii, T., Higuchi, T., 2002. Droplet formation in a microchannelnetwork. Lab on a chip 2 (1), 246.

[13]T. Ménard, S. Tanguy, and A. Berlemont. Coupling level set/vof/ghost fluid methods: Validation and application to 3dsimulation of the primary break-up of a liquid jet. International Journal of Multiphase Flow, 33(5):510 - 524, 2007.

[14]Mark Sussman and Elbridge Gerry Puckett. A coupled level set and volume-of-fluid method for computing 3d andaxisymmetric incompressible two-phase flows. Journal of Computational Physics, 162(2):301 - 337, 2000.

[15]Ronald P Fedkiw, Tariq Aslam, Barry Merriman, and Stanley Osher. A non-oscillatory eulerian approach to interfaces inmultimaterial flows (the ghost fluid method). Journal of Computational Physics, 152(2):457 - 492 , 1999. 
[16]Murray Rudman. A volume-tracking method for incompressible multifluid flows with large density variations. International Journal for Numerical Methods in Fluids, 28(2):357-378, 1998.

[17]G. Vaudor, T. Ménard, M. Doring, W. Aniszewski, A. Berlemont.A consistent mass and momentum flux computation method for two phaseflows. Application to atomization process. Computer and Fluids (submitted)

[18]M. Sussman, K.M. Smith, M.Y. Hussaini, M. Ohta, and R. Zhi-Wei. A sharp interface method for incompressible two-phaseflows. Journal of Computational Physics, $221(2): 469$ - 505, 2007. 